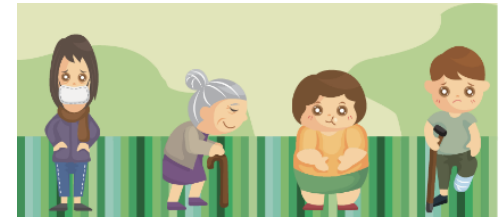

\title{
ANTE UN NUEUO PARADIGMA EN LA SALUD DE COLOMBIA
}

\author{
Juan Carlos González \\ Guillermo L. Restrepo Ch. §§§§§
}

Ensayo basado en la presentación Minsalud, Alejandro Gaviria, en el Congreso Nacional de Salud 2016

https://www.youtube.com/watch?v=k_aAvSu8rRg

Resulta muy evidente que, al menos en lo jurídico, Colombia está entrando a un nuevo paradigma en torno a la concepción del sistema de salud. La Ley Estatutaria de Salud (1) ha entrado en vigor y el Gobierno ha empezado a expedir los respectivos decretos buscando su implementación. Nosotros en Colombia hemos creído que los problemas necesitan cambios jurídicos para solucionarse, opinión que está muy bien expresada en todas las áreas y, la salud, no es la excepción. El informe de la OPS (2) respalda lo anterior al mencionar cuáles son los cambios primarios que deben tener los sistemas de salud para alcanzar la Atención Primaria en Salud (APS) en los países.
Convencidos de que la salud es el bien más preciado del ser humano (paradojalmente es cuando la perdemos que lo reconocemos y, cuando ello ocurre, el sistema de salud del país se convierte en algo clave pues es la única oportunidad que tiene la mayoría de la población para enfrentar esa desgracia), es que hemos considerado como un deber, contribuir al entendimiento del sistema de salud colombiano con sus problemas y con el planteamiento de soluciones, creyendo que así será más fácil comprometernos.

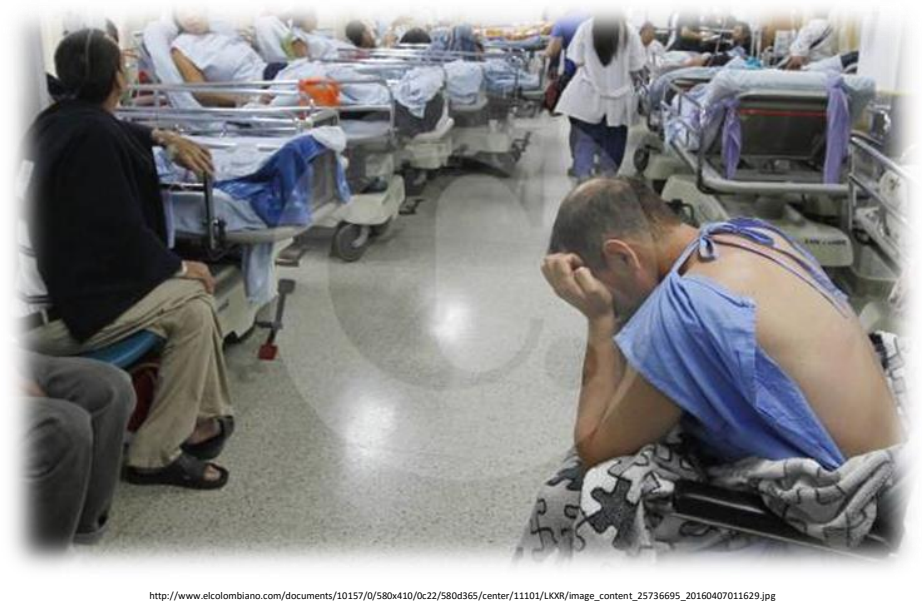

Colombia está viviendo una severa crisis del sistema de salud (3) y, no de ahora; nos atrevemos a plantear que es muy evidente desde 2009 (4) y la imagen que se observa la expresa muy bien. La sobrecarga de los servicios de urgencia, el sufrimiento y aparente abandono del paciente en un corredor atiborrado de otros pacientes; los familiares de aquel otro paciente asumiendo parte del cuidado (tal vez el consuelo) y, por el pasillo, un prestador de bata blanca (de seguro un médico) haciendo lo que mejor puede en la atención de la concurrida espera.

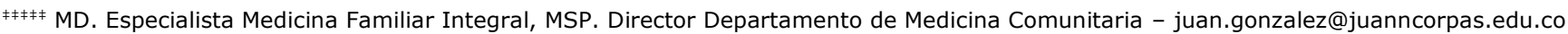
§§§§§ MD MPS. Director Postgrado Gerencia en Salud. FUJNC - Guillermo.restrepo@juanncorpas.edu.co 


\section{La APS}

El país ha escogido a la APS como la meta del modelo de salud que anhela (5). Este gobierno, casi desde sus inicios, orientó sus esfuerzos en tal sentido. Ha extendido una oportunidad inmensa con la continuidad que ha tenido el
Ministerio de Salud y Protección Social a través del último ministro, Alejandro Gaviria, quien con su equipo humano, ha mostrado suficientemente que está llevando a Colombia en tal dirección.

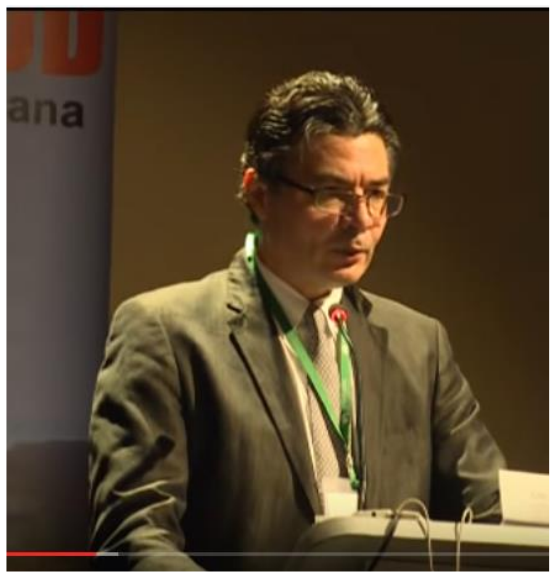

En noviembre de 2016, en el Congreso Nacional de Salud (6) el ministro hizo una muy interesante exposición acerca de cómo ve él la crisis de salud e hizo una serie de propuestas, que bien vale la pena analizar, buscando con ello cumplir nuestro objetivo, como es el contribuir al entendimiento de la situación. iTodos debemos entender el problema! Esto es un paso necesario para buscar soluciones.

\section{Una reflexión}

Afirma el ministro que antes de sepultar el Sistema de Seguridad Social, es importante reconocer uno de sus logros más significativos como es el haber sido un instrumento que ha permitido a Colombia alcanzar un punto muy alto en torno a la equidad.

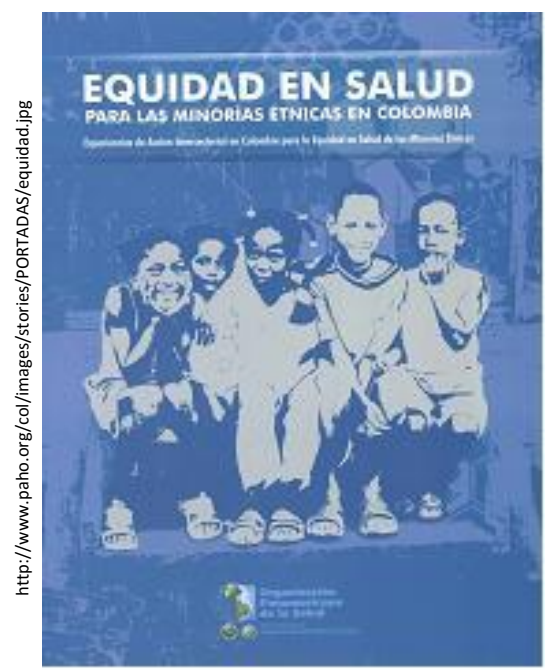

El desarrollo del régimen subsidiado justamente es, y ha sido, el logro. A través del aporte en proporción de lo que se recibe, y no aportar el que no recibe, Colombia logró ofrecer el acceso a la salud al menos a un sector grande la población, siendo, según el ministro, un ejemplo para el mundo. Al respecto, compartimos el sentir del ministro. En Colombia se logró vincular casi a toda la población a través de los dos regímenes y ciertamente los que tienen menos recursos reciben una atención aproximadamente igual a los que tienen más. 


\section{Pero...}

Sin demeritar tal logro, reconociendo que el acceso a situaciones graves se ha alcanzado, la realidad nos muestra que la atención no es buena en el sentido del trato y no lo es para ninguno de los dos regímenes.

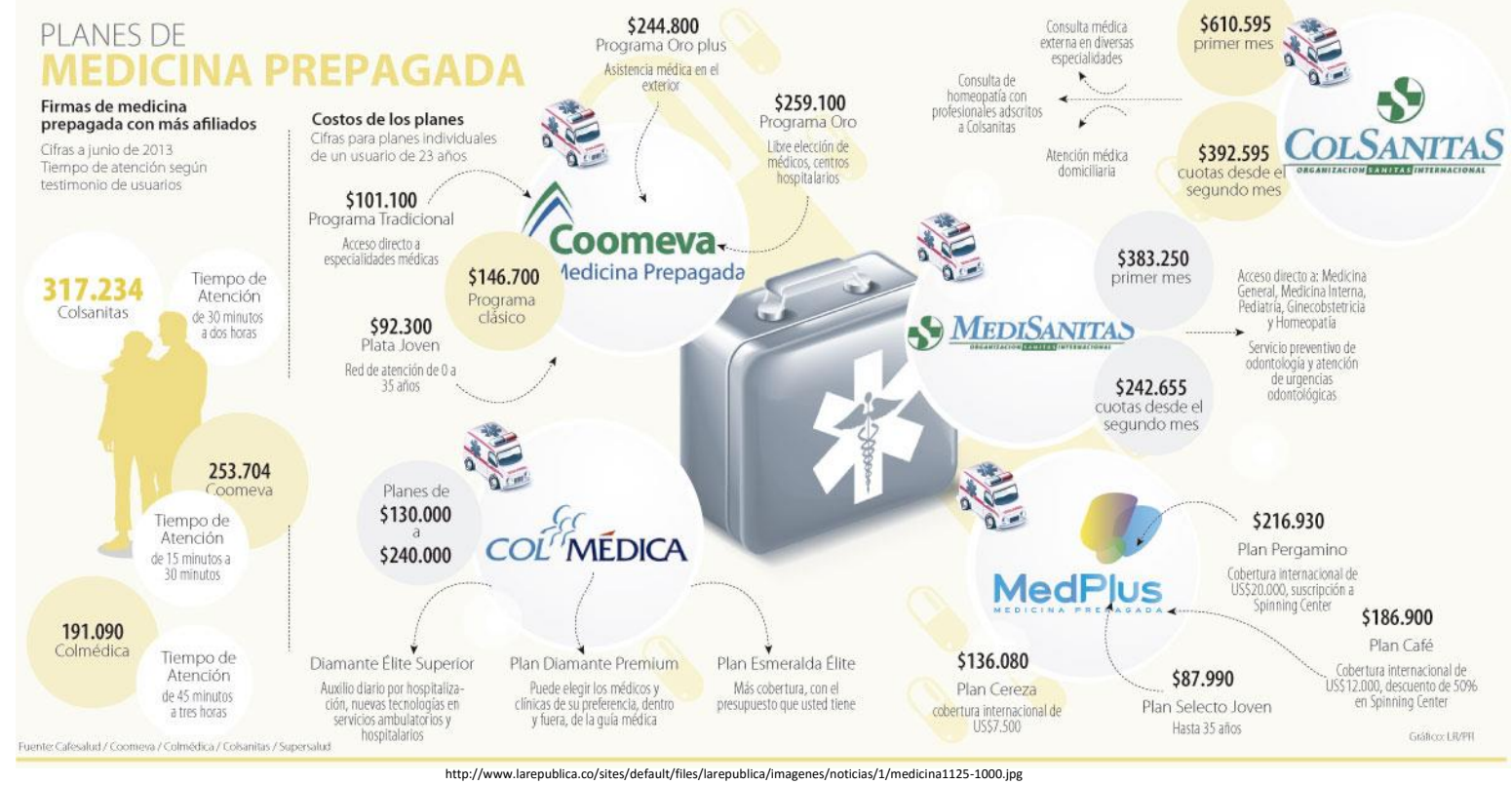

Esta situación ha dado pie a que florezcan las medicinas prepagadas, situación que violenta la equidad en torno a la salud, pues permite ver que aquellos que cuentan con más recursos pueden recibir una mejor atención. ¿Se puede ofrecer un trato humano y especial a través del sistema de salud de un país? Nosotros creemos que sí (7) y precisamente un modelo de atención basado en la APS puede ser la clave, donde la continuidad genere compromiso y confianza tanto por parte de los prestadores como de los pacientes.

Sin embargo, y reiterando el sentir del ministro, muchos de nosotros pudimos vivir la limitación inmensa de recursos para atender a la población más pobre del país antes de la década de los años 1990 y reconocemos que tal situación cambio mucho en los últimos 25 años.

\section{La crisis económica}

El ministro Gaviria sostiene que el problema del Sistema de Salud es una crisis económica con dos componentes distintos pero sumados.

1. La deuda acumulada.

2. El desequilibrio entre los gastos y los ingresos (causa de las causas).

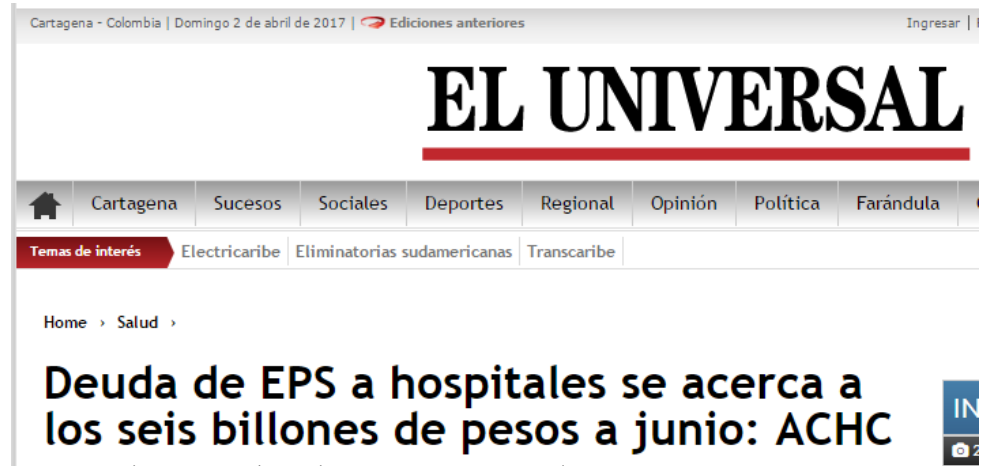

COLPRENSA | @EIUniversalctg | Bogotá | 5 de Noviembre de $201504: 41$ pm | 
Reconoce las deudas acumuladas, originadas, por un lado, por las atenciones no POS que el Gobierno (municipios) ha adquirido con las EPS (Empresas Promotoras de salud) y, a su vez, las que estas han adquirido con las IPS (8). ¿Cómo se producen? La dinámica es simple. Las IPS prestan la atención bajo dos premisas. La primera es la obligación de atender todo lo que les llegue por urgencia (deber) y, la segunda, es la "seguridad" de que las EPS les cancelarán. La confianza es la clave. Sin embargo, tal confianza se perdió con Saludcoop y Caprecom (ejemplo máximo), que dejaron a varias IPS con inmensas deudas, las que ahora mismo no saben si tal cartera se recuperará. Pero, y es lo grave, varias EPS han continuado con tal práctica. Afirman ellas que lo hacen porque no pueden pagar y no pueden porque los recursos que tienen no les alcanzan. Es así como en la contratación de las IPS con las EPS, se propone de un pago a 30 días de pasada la cuenta (lo que viene a ser dos meses, pues en el primer mes se causa la atención y en el otro mes se tramita la cuenta), pero la realidad es que tal pago se efectúa, en muchos casos, a 180 días (6 meses).

\section{Corrupción}

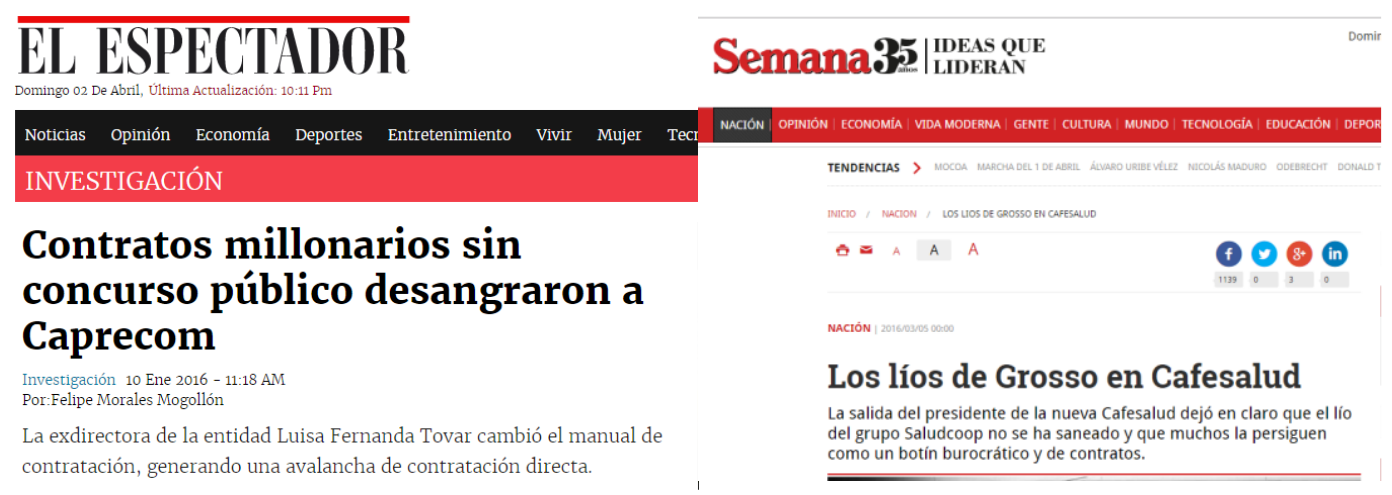

A ello es importante sumarle la corrupción (9) que surge, por ejemplo, con una desafortunada práctica implementada en el sector como es la de las "mordidas" en la contratación (contratación mentirosa o la práctica de "yo le pago ya, pero debe descontarnos un porcentaje").

\section{La causa de las causas}

Siendo lo anterior un problema muy importante porque tiene a muchas IPS en una situación de incertidumbre financiera que compromete su futuro, el ministro Gaviria afirma que el problema fundamental es que en el momento actual el
Sistema de Salud está gastando más de lo que recibe. Dijo en el encuentro que por cada 100 pesos que las EPS reciben, están gastando 104, es decir, existe un desequilibrio en el Sistema de Salud (10).

\section{EL NUEVO SIGLO}

\section{Noticias}

Desequilibrio de ingresos afecta la salud

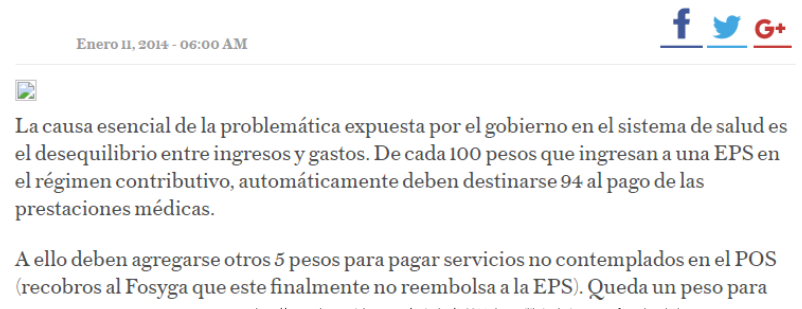

Afirma el ministro que esta situación se ha presentado porque hemos hecho un contrato incoherente. No tiene un futuro una situación en donde gastamos más de lo que ponemos. Prueba de las incoherencias es que la situación del Sistema de Salud tiene un problema con la gobernanza, pues se planea desde el Ministerio, pero la legislación de la Corte Constitucional no la tiene en cuenta y ordena gastos derivados de atenciones particulares y especiales. Pone de manifiesto lo que él considera abusos dentro del sistema; por ejemplo, la entrega de 500 pañales mensuales a ciertos pacientes o las atenciones fuera del país. 


\section{La presión tecnológica}

\section{SALUD}

\section{El precio de la insulina se triplica en seis años, de 200 a más de 600 euros al año por paciente}

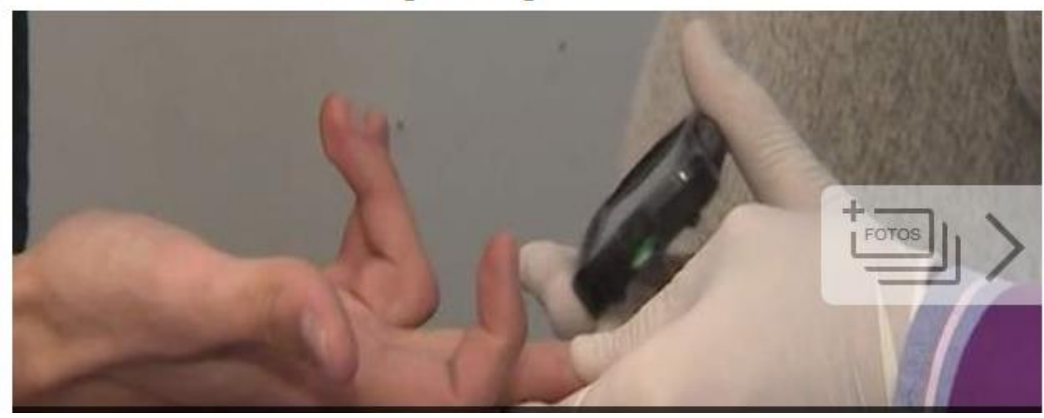

http://www.20minutos.es/noticia/2714577/0/precio-gasto-insulina-triple/ultima-decada-ano-paciente/

Dice que otra situación alarmante, y prueba de esa incoherencia, es la presión tecnológica y pone como ejemplo el manejo de la diabetes, el cual está basado desde hace más de 80 años, en buena parte, en el uso de la insulina. Pero, contrario a lo esperado, el costo de las mismas va en ascenso y todo por la salida al mercado de nuevos tipos que, si bien tienen mejoras evidentes, estas son marginales desde el punto de vita de la salud pública.

\section{Una política farmacéutica}

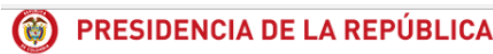

NUEVOPAIS

C

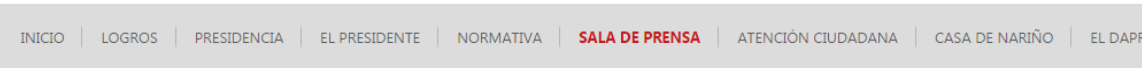

\begin{tabular}{|l|}
\hline TODAS LAS COLUMNAS \\
\hline PRESIDENCIA DE LA REPÜBLICA \\
\hline DELEGACIÓN DEL GOBIERNO \\
\hline $\begin{array}{l}\text { MINISTERIO DE AMBIENTEY } \\
\text { DESARROLLO SOSTENIBLE }\end{array}$ \\
\hline $\begin{array}{l}\text { MINISTERIO DE COMERCIO. INDUSTRIAY } \\
\text { TURISMO }\end{array}$ \\
\hline MIIISTERIO DE DEFENSA NACIONAL \\
\hline MINISTERIO DE EDUCACIÓN NACIONAL \\
\hline MINISTERIO DEL INTERIOR \\
\hline MINISTERIO DE MINAS Y ENERGIA \\
\hline MINISTERIOO DE SALUD Y DE LA \\
PROTECCION SOCIAL
\end{tabular}

$\mid \mathrm{NICIO}>$ Columnistas > Innovación, precios de medicamentos y desarrollo

- Columnistas

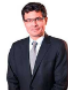

Innovación, precios de medicamentos y desarrollo

por Alejandro Gaviria Uribe, Ministro de Salud y de la Protección Social

Colombia, como muchos otros paises, tanto desarrollados como en desarrollo, ha definido la salud como un derecho humano fundamental, lo que significa, en esencia, que el acceso a los servicios de salud y a los medicamentos esenciales es una obligación del Estado y una cuestión de justicia y dignidad humana.

Los objetivos de desarrollo sostenible señalan de manera explicita que todos los Estados, sin excepción alguna, deben avanzar hacia "la cobertura sanitaria universal, en particular la protección contra los riesgos financieros, el acceso a servicios de salud esenciales de calidad y el

"El Ministro de Salud explica por qué el acceso a los medicamentos es una obligación del Estado y una http://es presidencia gov,co/columnas/Paginas/Innovacion-precios-de-medicamentos-y-desarrollo. aspx

Sostiene el ministro que el Estado debe imponer una política de control y regulación de precios a la industria farmacéutica con el fin de garantizar el acceso de los medicamentos a la población que lo requiera.

\section{Las soluciones}

Ante este panorama, el ministro habló de las soluciones que él ve como viables. 
$\rightarrow$ En torno a las deudas adquiridas:

En torno a las deudas adquiridas, se han otorgado créditos para aliviar las deudas de las EPS y de las IPS con el fin de que puedan sostenerse (11). Afirma el ministro que se han creado una serie de mecanismos jurídicos para asegurar que el problema de las deudas no se vuelva a presentar.

Considera que depurar a las EPS resulta clave. No cree que las EPS sean responsables, es más las considera necesarias dado que tienen conocimiento y experiencia.
Plantea que si, por ejemplo, las EPS se terminaran y las autoridades municipales asumieran la situación, no cree que eso cambiara en algo la situación actual. No lo cree pues explica que el problema no está en dichas entidades sino en el "desequilibrio del contrato social". Cree que, por el contrario, las EPS son mejores administradoras que el mismo Estado.

Considera indispensable la venta de CAFESALUD EPS, asegurando que, quien la compre, debe cancelar las deudas adquiridas y devolver la confianza.

\section{Lo de fondo}

Pero la forma que propone para enfrentar el desequilibrio del contrato social, ese gastar más de lo que recibe, la basa en la implementación de la Atención Primaria en Salud a través de la Política de Atención Integral de Salud (12), así como en una política farmacéutica (13) y, ciertamente, en incrementar los recursos del sistema (con impuestos a bebidas azucaradas, por ejemplo).

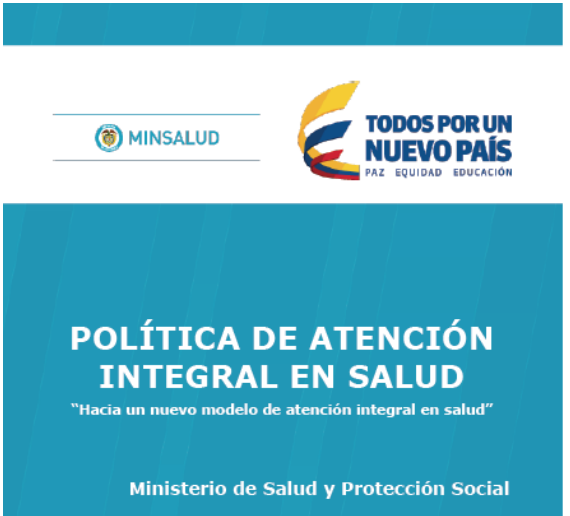

El desarrollar un modelo de atención cercano a la población probablemente sea una de las mejores formas de enfrentar la causa de las causas. El fortalecer la prevención y la promoción es la única forma segura de enfrentar a la enfermedad futura. Es, por decirlo de alguna forma, apostar

\section{Contexto institucional y justificación}

Sistema de sallud, ley estatutaria y pllan de desarrollo

\section{Pollíti:cal de attencilón inntegrall en} salduld a las bases, es decir, a la unión entre comunidad y prestadores y que desde allí se "cambie" el panorama incoherente de la salud actual. Implica enfrentar con coherencia esos gastos generados por las enfermedades de alto costo, siendo un buen ejemplo la política farmacéutica. 


\section{Transparencia}

Propone el ministro que el Gobierno y la ciudadanía debemos comprometernos con la transparencia.

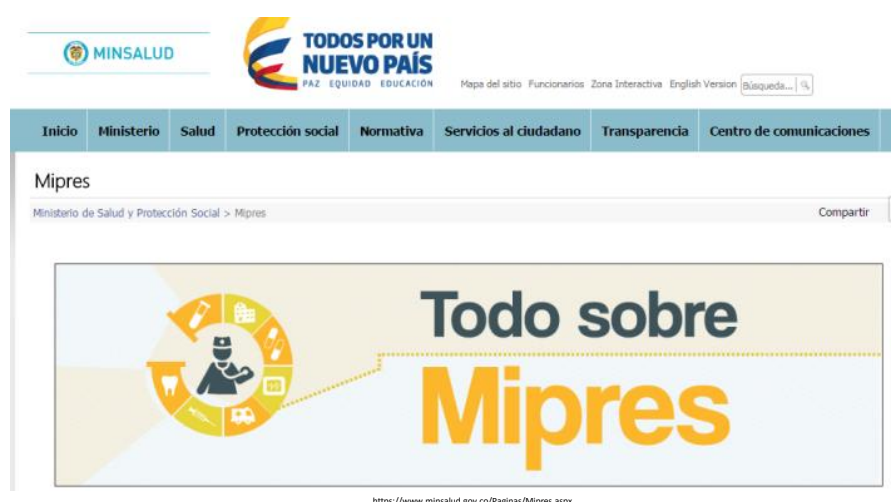

Un ejemplo es el uso de Mipres (14) en donde, respetando la autonomía del médico, se le hace responsable del buen uso de

la prescripción a través del uso de dicha plataforma que permite verificar la pertinencia del actuar del médico.

\section{Uso racional de las nuevas tecnologías}

Afirma que tenemos un reto en donde, entendiendo que existen avances científicos que permiten cambiar el pronóstico de enfermedades hasta antes improbables de curar, son habitualmente costosos y, por ende, se deberá crear un mecanismo que permita su financiación evitando que esto altere el equilibrio necesario del sistema de salud. ¿Cuáles o cómo? Ese será el reto del diseño.

\section{Ante todo esto}

Resulta evidente que el país se está dirigiendo hacia la APS.

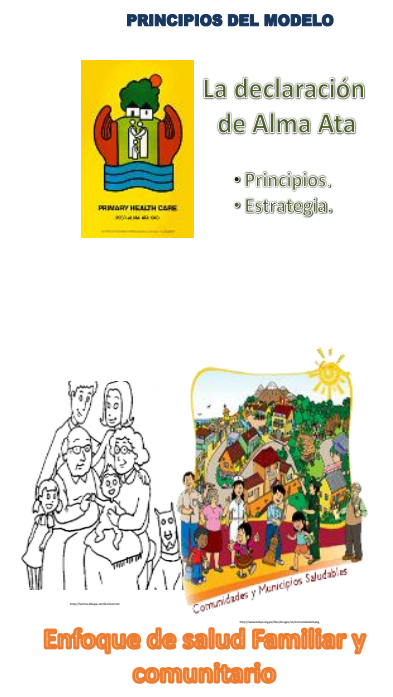

PRINCIPIOS DEL MODELO

La declaración

Principios

Estrategia
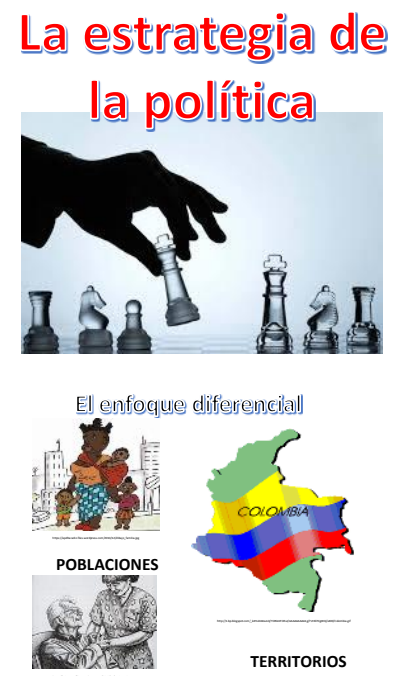

El cuiddado dẹ la salud

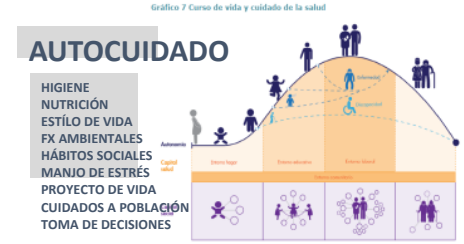

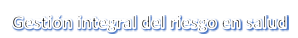

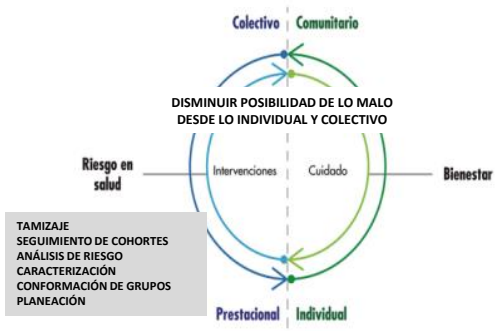

Fuente: MSPS. 2015. 
La ley estatutaria y el modelo de integración de atención en salud así lo dejan ver y esto, en sí mismo, implica un enorme cambio en la atención actual. Queda claro que, de no modificar la tendencia hasta hoy, de seguir atendiendo la enfermedad sin preocuparnos con fuerza y decisión por la prevención, se augura la quiebra del Sistema, en realidad, de cualquier sistema.

El ministro insiste en la necesidad de las EPS. Nosotros no las vemos. ¿Acaso las IPS no cuentan con un grupo de administradores que perfectamente se pueden hacer cargo, como hoy lo hacen, de las cuentas de la prestación? ¿Qué necesidad se tiene de duplicar estas actividades? ¿Acaso hoy no conocemos el costo de las atenciones con lo que, contratar de forma justa, es relativamente sencillo? Pero, y además, basados en la experiencia, ¿cuál es o cuál ha sido el aporte de las EPS al Sistema?

¿No será posible concebir la atención a través esas redes integradas de salud que propone la APS? ¿Por qué no dejar que esas redes se organicen junto con la población sin necesidad de las EPS como intermediarias?

\section{Un análisis}

En el número 137 de la Carta Comunitaria (marzo-abril de 2016; Vol. 24 No 137), nos referimos a ello y, por lo mismo, transcribimos:

\section{EL ERROR: CREER QUE SE PUEDEN UNIR ENTIDADES CON OBJETIVOS DIFERENTES}

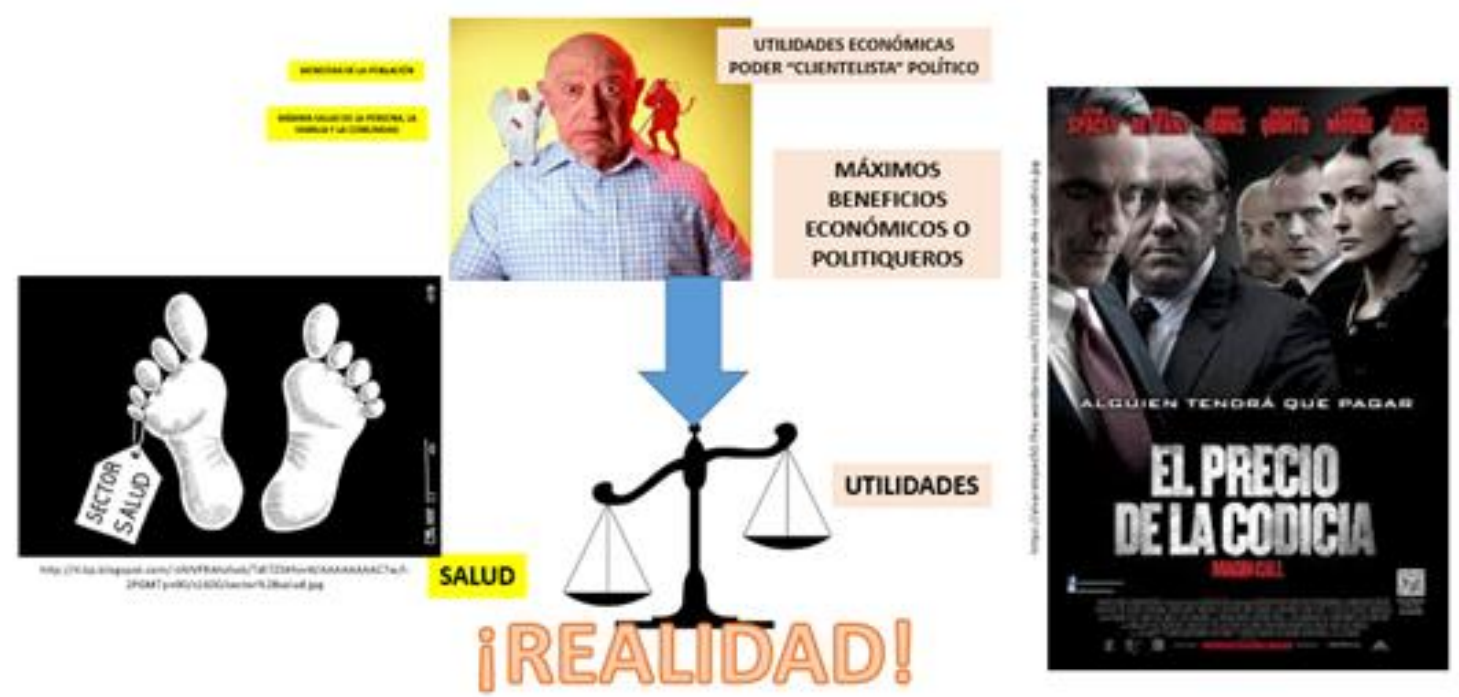

Sostenemos que el principal problema del actual sistema de salud es creer que entidades con objetivos tan disimiles se puedan unir para servir a la población.
Son tres los entes que han de coordinar la política de atención integral en salud de la población; EPS, el ente territorial y las redes integradas, y sostenemos que "unirlas" es un esfuerzo inútil.

\section{Semana35 $\left.\right|_{\text {LIDERAN }} ^{\text {IDEAS QUE }}$}

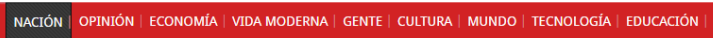

TeNo

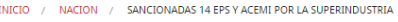

$$
\therefore=\text { A A A }
$$

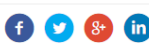

\section{Sancionadas 14 EPS y ACEMI por} la Superindustria

El caso, que se conoció como el 'Cartel de la EPS', se hizo público en marzo de este año, el mismo mes en que estalló el escándalo del
Sostenemos que el principal problema de la seguridad social se desprende del claro interés que tienen las EPS en torno a "ganar" utilidades desde el punto de vista económico; que no es la salud de la población y que, para ganar, incluso el ponerse de acuerdo para hacer trampa puede ser reconocido como una supuesta "buena práctica" (15). La evolución de estos 25 años de la seguridad social proporciona suficiente evidencia para comprobar cómo, usando los recursos, una buena cantidad de EPS se dedicaron a hacer "negocios" (16), dejando en un nivel secundario la salud de la población.

La práctica política en torno a la salud en estos últimos 25 años ha tenido como principal objetivo la adquisición de poder burocrático y clientelista y no la salud de la población. En tal sentido, tan solo los prestadores tendrían el interés de brindar el mejor servicio a la población, ello a pesar de la corrupción que también ha invadido a parte de este sector en donde, además, la explotación a los prestadores primarios se ha convertido en la norma antes que en la excepción. 


\section{Una realidad}

Como de esos dos sectores (EPS y autoridades municipales), es el ente territorial el que debe permanecer por nuestro modelo político, una primera propuesta es eliminar a las EPS del sistema y, en torno al ente territorial, poner a sus funcionarios bajo el rango de carrera administrativa, con lo cual se anhela que la salud sea dirigida y ejecutada por un perfil técnico con las ventajas que de allí se pueden desprender.

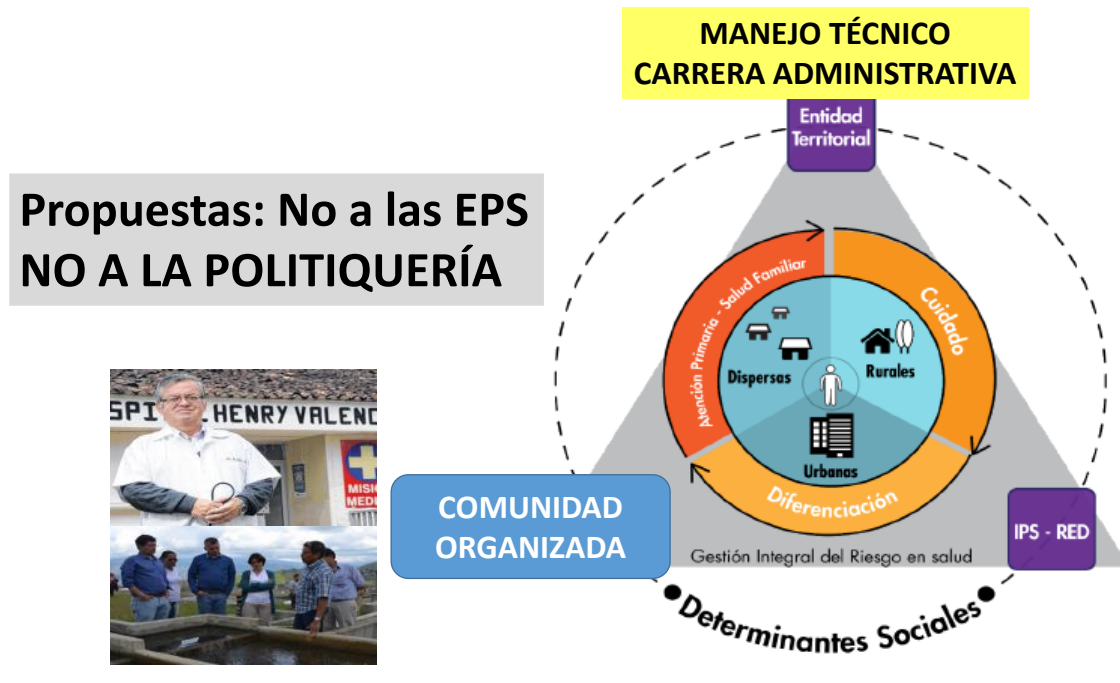

Creemos que ha de ser la comunidad organizada la que entra a ser esa parte de coordinación y evaluación del modelo. Experiencias como la desarrollada en Versalles (Valle) ponen de manifiesto lo que la comunidad organizada puede hacer en torno a la salud y otros aspectos. El Ministerio precisamente publicó un documento (17) de esta experiencia (y otras) refiriéndose a ella como exitosa.

\section{La clave: las redes integradas de salud}

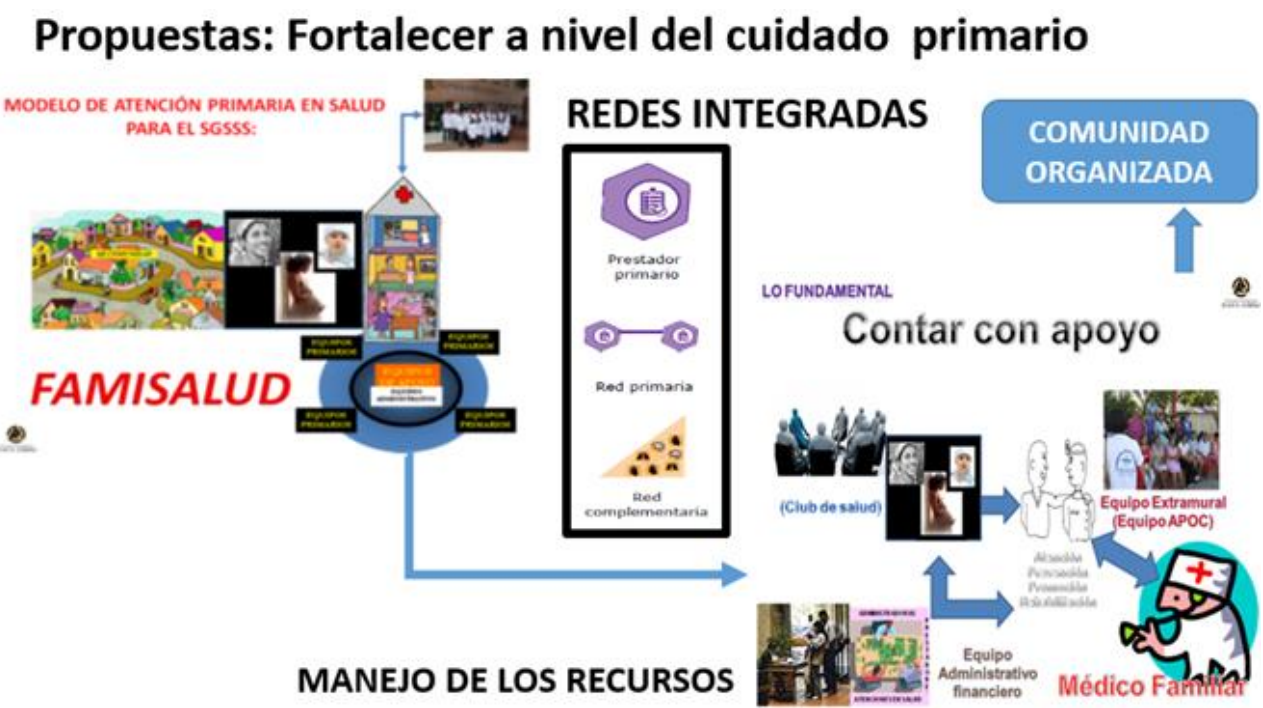

La clave del éxito de la política de integración está en el cuidador primario. Eso queda reflejado en estos sencillos puntos: 
1. Que la población pueda acceder a un centro de salud cerca de su casa donde sea reconocida y atendida por un equipo comprometido y que cuente con la capacidad de resolver los principales problemas del paciente o pueda recibir apoyo cuando lo requiera, por ejemplo, del médico familiar.

2. Tal centro de salud tendrá unos equipos extramurales capaces de penetrar en el barrio y de identificar los procesos comunitarios existentes, lo que contribuirá a organizar y vincular al centro con el fin de ofrecerle a esa comunidad la mejor atención.

3. Ese equipo de salud será el responsable de caracterizar a la población a su cargo.

4. Desde este centro se construirán las rutas integrales, asumiendo la ruta del mantenimiento de la salud, incluyendo en esta las acciones de salud pública que se puedan ofrecer desde este nivel de atención.

5. Asimismo, y a través de la caracterización, construirá los planes para los grupos de riesgo, dejando bien definidas las rutas en donde incluirá las valoraciones por los especialistas cuando se requieran.

6. Cuando se requiera la hospitalización de algún paciente, el cuidador primario, a través de la ruta, sabrá remitir al hospital de la red donde pueda recibir la mejor atención (ideal que sea cercano), enviando toda la información pertinente.

7. El cuidador primario (el centro de salud) tendrá un equipo encargado de penetrar el barrio e identificar los riesgos y hacer planes de intervención a través, incluso, de visitas a la vivienda (ofrecerle las acciones de salud pública).

8. Con base en el conocimiento de la zona, promoverá u organizará a la comunidad, la cual se convertirá en participante de los procesos en torno al autocuidado, pero, además, en evaluadora de la forma como logra preservar su salud y la forma como es atendida en toda la red. Los miembros de los equipos de salud deben, en lo posible, vivir en esas comunidades.

9. Dado que el compromiso y la continuidad entre prestadores y comunidad es clave, el prestador debe trabajar sabiendo que ese es su proyecto de vida laboral, con unas condiciones tales que sienta que está en el mejor sitio para trabajar.

10. El prestador deberá tener acceso a un programa de educación continuada que busque, por encima de todo, el bienestar de la población que atiende.

\section{El uso de los recursos}

Sostenemos que para este momento el país ya tiene cifras de los costos de la prestación, por ende, el giro hacia las redes integradas puede hacerse de forma más o menos igual y bien calculada, sabiendo cuántos recursos necesitará el cuidador primario, así como los complementarios. El cuidador primario debe:

$\rightarrow$ Elaborar las rutas de atención.

$\rightarrow$ Gestionar la prestación de los servicios a nivel individual.

$\rightarrow$ Establecer, gestionar y evaluar la acción con los grupos de riesgo.

$\rightarrow$ Manejar contablemente el presupuesto de toda la red de servicios y ser el ordenador del gasto.

$\rightarrow$ Contratar con el nivel complementario la prestación de los servicios para los niveles superiores (las redes se deben formar de abajo para arriba).

$\rightarrow$ Establecer una comunidad de servicios con su red.

$\rightarrow$ Realizar las consultas de las especialidades básicas definidas para cada territorio, tanto a nivel médico como paramédico y recibir la hospitalización correspondiente a patologías de mediana complejidad.

$\rightarrow$ Manejar las redes de remisión, contrarremisión e interconsultas.

$\rightarrow$ Elaborar el plan de salud para el municipio.

$\rightarrow$ Integrar la promoción, la prevención, la recuperación y la rehabilitación, tanto a nivel individual como colectivo, incorporando todas las actividades de salud pública. 


\section{El equipo de los cuidadores primarios}

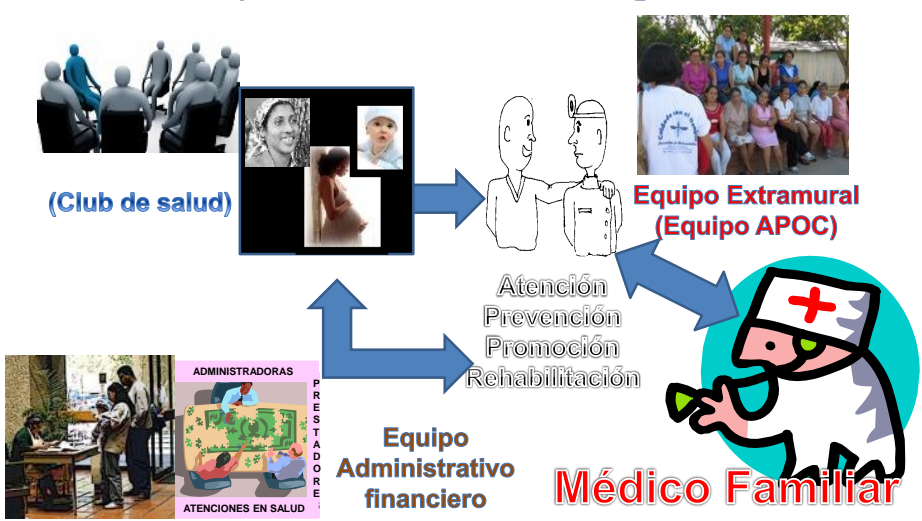

Estará constituido por médicos generales, auxiliares de enfermería, agentes comunitarios y comunidad organizada, acompañados por médicos familiares, enfermaras y odontólogos. Sus sitios de trabajo serán los puestos de salud, los centros de salud y los hospitales locales. Responderán por:
- El diagnóstico de las familias asignadas.

- La atención integral.

- La atención de la salud comunitaria, entendida como el apoyo a los sitios donde está la comunidad tales como colegios, guarderías o grupos de adultos mayores.

\section{Lo cierto es que...}

Un nuevo sistema de salud se está gestando en Colombia el cual está impregnado de Atención Primaria en Salud. Analizar las razones por las cuales estamos en la crisis de salud actual resulta fundamental para que las propuestas que surjan, corrijan debidamente los problemas detectados. Ese ha sido el objetivo de este ensayo resumen.

Para nosotros, desde nuestra facultad de Medicina lo que está sucediendo es una inmensa oportunidad de aportar y comprometernos con nuestra razón de ser y con el país.

\section{REFERENCIAS}

(1) Ley estatutaria. Disponible en: https://www.minsalud.gov.co/Normatividad_Nuevo/Ley\%201751\%20de\%202015.pdf

(2) Organización Mundial de la Salud [Internet]. Informe sobre la salud en el mundo. Disponible en: http://www.who.int/whr/2008/summary/es/

(3) El colombiano [Internet]. Crisis de la salud en Colombia. Disponible en: http://www.elcolombiano.com/colombia/crisis-de-la-salud-en-colombiaEE3897868

(4) Montoya M. Revista electrónica, facultad de derecho y ciencias políticas, U de A, número 3, año 1, enero abril de 2010 . Disponible en https://aprendeenlinea.udea.edu.co/revistas/index.php/derypol/article/.../5144/4505

(5) Minsalud. Documento marco de la Política de la Estrategia de Atención Primaria en Salud en Colombia. Disponible en: https://www.minsalud.gov.co/salud/Paginas/Consultas-Estrategia-de-Atencion-Primaria-en-Salud-en-Colombia.aspx

(6) Minsalud. Gaviria A. Congreso Nacional de Salud, 2016. Disponible en: https://www.youtube.com/watch?v=k_aAvSu8rRg

(7) González JC, Restrepo G, Hernández AD, Ternera DC, Galvis CA, Pinzón JA. Satisfacción de pacientes que acudieron al primer nivel de atención en Bogotá. Rev salud pública [Internet]. 2014 Dic [citado 2017 Abr 01]; 16(6): 871-884. Disponible en:

http://www.scielo.org.co/scielo.php?script=sci_arttext\&pid=S0124-00642014000600006\&lng=es. http://dx.doi.org/10.15446/rsap.v16n6.38192.

(8) El Universal [Internet]. Deuda de EPS a hospitales se acerca a los dos billones de pesos a junio: ACHC [5 de noviembre de 2015]. Disponible en: http://www.eluniversal.com.co/salud/deuda-de-eps-hospitales-se-acerca-los-seis-billones-de-pesos-junio-achc-210355

(9) Semana [Internet]. Los líos de Grosso en Cafesalud [5 de marzo de 2016]. Disponible en: http://www.semana.com/nacion/articulo/saludcoopsalida-de-grosso-de-cafesalud-revelaria-corrupcion/464062

(10) El Nuevo Siglo [Internet]. Desequilibrio de ingresos afecta la salud [11 de enero de 2014]. Disponible en: http://www.elnuevosiglo.com.co/articulos/1-2014-desequilibrio-de-ingresos-afecta-la-salud

(11) Minsalud [Internet]. EPS e IPS han recibido créditos de Findeter por cerca de medio billón de pesos [5 de febrero de 2016 ]. Disponible en: https://www.minsalud.gov.co/Paginas/EPS-e-IPS-han-recibido-creditos-de-Findeter-por-cerca-de-medio-billon-de-pesos.aspx

(12) Minsalud [Internet]. Polítia de Atención Integral en Salud. Disponible en: https://www.minsalud.gov.co/sites/rid/Lists/BibliotecaDigital/RIDE/DE/modelo-pais-2016.pdf

(13) Documento Conpes Social. Consejo Nacional de Política Económica y Social. República de Colombia. Departamento Nacional de Planeación [30 de agosto de 2012]. Disponible en: https://www.minsalud.gov.co/Documentos\%20y\%20Publicaciones/Politica\%20Farmac\%C3\%A9utica\%20Nacional.pdf

(14) Minsalud [Internet]. Todo sobre Mipres. Disponible en: https://www minsalud gov co/Paginas/Mipres.aspx

(15) Semana [Internet]. Sancionadas 14 EPS y ACEMI por la Superindustria [1 de septiembre de 2011]. Disponible en: http://www.semana.com/nacion/articulo/sancionadas-14-eps-acemi-superindustria/245800-3

(16) Semana [Internet]. El negocio de la salud [20 de febrero de 2012]. Disponible en: http://www.semana.com/opinion/articulo/el-negociosalud/253735-3

(17) Análisis y propuesta para el desarrollo de la Atención Primaria, la Promoción de la Salud y el enfoque de determinantes orientados a la reducción de las inequidades, articulado al sistema de salud colombiano. INFORME FINAL. 14 de septiembre de 2012. 